3. Review Reports of the Guatemala-Japan Cooperative Project on Onchocerciasis Research and Control

\title{
1) Guatemala-Japan Cooperative Project on Onchocerciasis Research and Control: A Review of the Technical Cooperation, Case Study Series No. $6^{*}$
}

\author{
Kunitoshi Sakurai
}

This project achieved remarkable success and results in the field of medical cooperation for the following reasons.

First, it was a project that required multiple methodologies not seen in any previous medical care cooperation project, extending over fields such as epidemiology, parasitology, entomology and ophthalmology. Successful integrations of various fields were considered necessary.

Secondly, as an effective chemotherapy was not yet established for onchocerciasis, the blackfly control was considered the principal measure. In Guatemala, the vector blackfly breeds in innumerable small streams on the slopes of mountainous districts. Enormous outdoor work was expected necessary to attain the goal. As Japanese specialists were not experienced in such large-scale fieldwork to eliminate the vector blackfly, the feasibility of the project was a subject of controversy.

The third factor in the success of the project was the collaboration of fundamental research and tactics mainly on the Japanese side and the experience of vector control mainly on the Guatemalan side.

The National Malaria Eradication Service (SNEM) of Guatemala, the body in charge of the blackfly control trials in this project, had longstanding experience in mosquito control and a trained and talented staff. The outstanding efforts of SNEM to control the blackfly were supported by the fundamental research and tactics developed by the Japanese specialist team. Here we see an ideal form of cooperation in a project conducted by counterparts in two countries.

March 30, 1985

\section{QUOTATION SOURCES}

1) A specialist report by Isao Tada, Feb., 1974. Dept. Med. Coop. Medicine-73-14-(104)

2) A JICA report (Basic Investigation Team), June, 1975. Dept. Med. Coop. Medicine-74-39 (129)
3) A JICA report (Implementation Investigating Team), Aug., 1975. Dept. Med. Coop. Medicine-75-7 (136)

4) A JICA report (Programming Team), Nov., 1977. Dept. Med. Coop. Medicine-77-9 (172)

5) The first project report (Onchocerciasis), Mar., 1978. Dept. Med. Coop. Medicine-78-2 (186)

6) The second project report (Onchocerciasis), Mar., 1979, 2nd Dept. Med. Coop. Medicine-2 -JR-79-1

7) The third project report (Onchocerciasis), Mar., 1980, 2nd Dept. Med. Coop. Medicine-2-JR-80-24

8) A JICA Project evaluation team report (Onchocerciasis), Mar., 1980, 2nd Dept. Med. Coop. Medicine-2JR-80-4

9) A JICA Project evaluation team report (Onchocerciasis), May, 1980, 2nd Dept. Med. Coop. Medicine-2JR-80-23

10) Proceedings of The Guatemala-Japan Joint Conference on Onchocerciasis Research and Control, 1981. Dept. Med. Coop. Medicine-JR-82-10

11) The fourth project report (Onchocerciasis), Apr., 1981, 2nd Dept. Med. Coop. Medicine-2-JR-81-21

12) A guidebook for Guatemalan onchocerciasis (Robles disease) — with special reference to vector control by T. Suzuki. Sep., 1983. JICA

13) Manual of Onchocerciasis (Robles disease) control in Guatemala, Sep., 1983. JICA

14) Glossary for Technical Terms on Onchocerciasis (Robles disease) in Guatemala, Sep., 1983. JICA

15) Working report (from May to Aug. 1984) by $T$. Suzuki, M. Ito and S. Ito, Sep., 14, 1984. JICA

16) General report by Y. Yamagata, Nov., 15, 1984. JICA

17) Evaluation report for the onchocerciasis research project in Guatemala at the end of the project, To be published in Mar., 1985

18) [Cooperation framework between the specialists and counterpart: An observation] by Y. Yamagata, International Cooperation (submitted)

\footnotetext{
*Reproduced and translated of the report JR. 85-37 with the kind permission of Institute For International Cooperation (IFIC), JICA.
} 


\section{Overview of the Onchocerciasis Study Project in the Republic of Guatemala}

\section{1-1 The background of requests for cooperation from Guatemala}

Onchocerciasis caused by the filaria Onchocerca volvulus is distributed over tropical Africa and parts of Central and South America. The number of patients is estimated to reach 20 million all over the world. Onchocerciasis is regarded to be next in importance to malaria by WHO. Microfilariae of the parasitic worm enter the human eye, causing inflammation and often loss of eyesight. The highest prevalence is observed in West Africa, and WHO is thus implementing large-scale control measures in seven countries including Upper Volta in view of the negative impact of blindness on the local socioeconomic development.

On the other hand, the existence of the disease has been observed in Mexico and five other countries in Central and South America, although control measures have not been implemented by any international organization even in the two countries with the greatest endemic areas, namely Mexico and Guatemala. The lack of control measures in Latin America is attributable to the difference in blackfly breeding places in water systems. In Africa, broad rivers are the breeding place for the vector blackfly, while in Mexico and Guatemala blackfly larvae breed in extremely small streams on mountain slopes. Elimination was considered very difficult in Central America.

In the Republic of Guatemala, however, coffee plantations are numerous in the mountainous endemic areas, and coffee is the main industry in the country. As a result, onchocerciasis was prevalent among plantation workers. Concerned about the impact of this situation, the government of Guatemala requested medical assistance from Japan (quotation source No. 8, p. 3).

\section{1-2 Paths of cooperation}

The medical cooperation project in the Republic of Guatemala started after the preliminary investigation (quotation source No. 1) conducted between November 1973 and January 1974. A basic investigation team was dispatched in March 1975 (quotation source No. 2) at the request of the Republic of Guatemala, and finally an implementation team was dispatched in June and July 1975 (quotation source No. 3). The R/D (record of discussion) was sealed on July 21 and the project started on Oct. $1,1975$.

Later the parties decided to conduct the project for three more years as part of a second phase implemented by the next R/D sealing (quotation source No. 9) on May 26,
1980, thus extending the cooperation to a total of eight years. The project ended on September 30, 1983.

\section{1-3 Purpose and method of the projects \\ (1) The final purpose}

To establish control measures for onchocerciasis in Guatemala and eventually other Central and South American countries.

(2) Aim

1) Choosing a pilot research area to implement control measures and to show the impact of the measures as a real result.

2) Assembling fundamental research and practical experience useful for the control measures.

3) Educating counterpart trainees capable of continuously implementing the control measures in the future.

(3) Methodology

1) The project period is five years

2) A tentative vector control is adopted as a control measure.

3) The methods to effectively assess control measures should be established in insects and humans over the long and short term to determine the appropriateness of the measures.

4) Fundamental research indispensable for the control measures should be carried out in the fields of epidemiology, parasitology and entomology.

5) Efforts should be made to dispatch specialists from Japan, obtain equipment and train counterparts in Guatemala on the basis of close cooperation between the two countries (quotation source No. 8, p. 4).

The cooperation project basically calls for the establishment of effective onchocerciasis control measures based on appropriate research and field trials and their implementation in the Republic of Guatemala. In other words, Japan does not aim to control by ourselves onchocerciasis in the Republic of Guatemala. Therefore, in addition to the establishment of control measures, the education of specialists and technical staff in the counterpart country must be the purpose of technical guidance and field training. In addition, the reorganization of the associated authorities and institutions in the counterpart country should be considered in order to ensure the appropriate use of the established control measures in the future (quotation source No. 3, p. 4).

\section{1-4 Character of the project and points requiring particular attention}

Approximately 20 project-type medical cooperation projects had been launched on the basis of Japanese government ODA as of 1975. Most involved a single dispatch 
of specialists to an educational institution and spot-type cooperation with a research organization or a specific section of a hospital.

In contrast, the present project covered a pilot area of $300 \mathrm{~km}^{2}$ and had a complex character involving various specialized fields, expanding from the conventional spottype cooperation to a two-dimensional project in order to establish endemic disease control measures.

In addition, as a trend in the field of international health, direct cooperation involving public health, endemic disease control and nourishment improvement etc. was considered important for the life and welfare of the people. In view of this fact, the present project was a pioneering endeavor in Japan's history of medical cooperation. The following difficulties and problems were expected to occur.

\section{(1) Hurdles faced in the extensive fieldwork}

1) Preparation of a water system map depicting several thousand streams covered with high grass in the pilot area including various plantations (finca), 2) Blazing of paths along these streams for insecticide spraying, 3) Dissemination of knowledge (PR) among the uneducated inhabitants living in endemic areas.

Small goals needed to be accomplished before the main project, mainly through the leadership of the Japanese side although the Guatemalan side originally had responsibility.

\section{(2) Coordination}

The cooperation project involved the fields of epidemiology, parasitology, entomology and ophthalmology and extended to Direction General, Robles Hospital, Main Laboratory (SNEM) and dozens of plantations lying scattered in the pilot area at the time of commencement. It was important to coordinate them by maintaining proper connections among the organizations in the project diagram.

\section{(3) Exclusive use of Spanish}

Only two people including $\mathrm{Mr}$. O. Ochoa, a trainee in Japan, were capable of interpreting for the Guatemalan staff and inhabitants in the field. The Spanish translation of reports was a high-priority issue that needed to be solved urgently in order to present results clearly.

\section{(4) WHO, PAHO, WB and other international organizations}

As a post-malaria issue, WHO had already started an onchocerciasis control program in African foci from 1970, also adopting the vector control strategy. However, the methodology for the effective measurement of vector control was not yet established.

Furthermore, anti-onchocercal medicines were yet to be developed. Thus the exchange of information was essential. In this situation, the above organizations showed a strong interest in this project because it focused on Central America-specific transmission. The U.S.A. and West Germany also had plans to launch bilateral research projects in Guatemala (quotation source No. 3, p. 41-43).

\section{1-5 Outline of the cooperation project}

During the eight years of cooperation, the cumulative number of dispatched specialists reached 90, including seven investigation teams and 27 specialists dispatched from Japan for consultations. Moreover, the Japanese side accepted 13 trainees and donated machines and equipment worth approximately 190 million yen to Guatemala.

The large number of dispatched specialists indicates that this was a large-scale project second only to the Traffic Center Project in the Philippines (quoted from p. 79 of "Ten years Progress of Japan International Cooperation Agency").

Celebrating the end of the first five-year phase of the cooperation project, an international congress on onchocerciasis research was held at the Hotel El Dorado, Guatemala City, for five days from January 12 to 16, 1981 (quotation source No. 10). The event provided a forum for the presentation and evaluation of all the scientific and technical findings obtained through the project in order to contribute to onchocerciasis control in Guatemala.

In the final stage of the extended project in September 1983, technical manuals regarding onchocerciasis control in Guatemala were published in Spanish and English, citing the findings obtained in the project (quotation source No. 13).

\section{1-6 Evaluation of the project}

In July 1983, an evaluation team was dispatched prior to the project expiration. The team engaged in discussions with the National Malaria Eradication Service (SNEM) in the Ministry of Health and Welfare, the operating organization in the Republic of Guatemala, as well as interviews with dispatched Japanese specialists and inspections of San Vicente Pacaya, the pilot area. The following is a list of the five general evaluations made.

(1) The project lasted for eight years from October 1975 and produced remarkable results in basic research and control technology, particularly blackfly control, of onchocerciasis in Central America.

(2) Meticulous field surveys made it possible to produce a detailed water system map and to spray insecticide (Abate) in the numerous target streams, covering roughly half of the pilot area where blackflies breed and transmit onchocerciasis. A marked reduction of the vector blackfly was observed in the sprayed areas. This result was encouraging and indicated the success 
of the control measures.

(3) Considering the successful experimental control, SNEM, the operating organization for malaria eradication in Guatemala, played a very important role in accomplishing such remarkable results.

(4) Japanese contributions in terms of specialist dispatch (total of 90), trainee acceptance (total of 13) and the donation of equipment (approximately 190 million yen) were indispensable in supporting the cooperation project.

(5) The results of the present project were hailed internationally at the "Japan-Guatemala Congress of Onchocerciasis" held in Guatemala in January 1981 (quotation source No. 17).

At this moment, effective and safe anti-onchocercal drugs remain unavailable. Vector control is the only way to interrupt the transmission of onchocerciasis. Accordingly, the purpose of the present project can be summarized by the following three points, differing slightly from those mentioned in 1-3.

1) Establishment of a Guatemalan method of vector control based on basic studies

2) Vector control trials in the pilot area

3) Evaluation of vector control by epidemiological indices in terms of the reduction of prevalence among inhabitants

Y. Yamagata, a JICA specialist remaining for followup studies after the project, pointed out that, at the initial stage of vector control trials, the success of vector control seemed improbable. Unlike Africa, the blackfly larvae breed in a mountainous environment in Guatemala, a condition that made it seem impossible to spray insecticide in all the breeding sites. Furthermore, none of the Japanese specialists had experience in insecticide spraying. However, a small-scale vector control trial showed success. In 1979 , the trial succeeded in diminishing the vector density to $1 / 100$ of the pre-control level in a $3 \mathrm{~km}^{2}$ pilot area. The trials were steadily expanded to $95 \mathrm{~km}^{2}$ by the end of the project and to $151 \mathrm{~km}^{2}$ in 1984 through the conscientious efforts of the Guatemalan staff. Unfortunately, since the disease is chronic in nature, epidemiological evidence of a reduction in transmission was not yet obtained (quotation source No. 18).

The final evaluation report (quotation source No. 17) concluded that the project was one of the most successful undertakings of JICA in the medical cooperation sector, accomplishing the initial target schedule steadily over the course of eight years.

The government of Guatemala expressed gratitude for the success of this JICA project. Other countries suffering from the disease, such as Mexico, Colombia, Venezuela,
Brazil and Ecuador, also showed a keen interest. WHO, which is now wrestling with vector control in Africa where an even higher prevalence is observed, applauded the outcome of the present project.

\section{Conclusion of R/D (Record of Discussion) and the CoOperation Policy}

\section{2-1 Process leading to the $R / D$}

On the basis of the preliminary investigation by Prof. Isao Tada (Kanazawa Medical University) from November 1973 to January 1974 (quotation source No. 1), an investigation team headed by Prof. Akira Nakajima (Juntendo University) traveled to Guatemala in March 1975 (quotation source No. 2), and JICA decided to develop effective onchocerciasis control measures in Guatemala through basic and applied research trials (quotation source No. 3, p. 4). Then in July 1975, Dr. Shigeo Hayashi (Dean, National Institute of Health) headed a project implementation team to join with Guatemalan authorities in discussing the content of cooperation and the implementation policy. On July 21, 1975, the R/D was signed and exchanged between Dr. Julio Castillo Sinibaldi (Minister of Health and Welfare), Guatemala and Dr. Shigeo Hayashi, Japan. The text of the $\mathrm{R} / \mathrm{D}$ is quoted in document No. 3 p. 27-34.

\section{2-2 Cooperation and implementation}

The contents of the medical cooperation in this project are the activities in the following four fields.

\section{(1) Epidemiology}

This sector covers epidemiology, parasitology and clinical research. Important baseline data before the control trial should be established and the pre-control prevalence of onchocerciasis determined. The method of evaluating the control operations should also be examined. Epidemiologists, parasitologists, ophthalmologists, clinicians, statisticians and office workers are assigned to this field.

\section{(2) Vector control}

Taxonomical, morphological, biological and physiological studies of blackflies are performed in this field. Furthermore, transmission potential, insecticide sensitivity, the insecticide application and evaluation measures in the blackfly are dealt with in this field. Another important task is the preparation of a detailed map showing the breeding sites of blackflies. Entomologists, insecticide specialists and map-makers are assigned to this field.

\section{(3) Treatment}

Onchocerciasis section in the Guatemalan staff has conducted a continuous nodulectomy campaign for the treatment of onchocerciasis, i.e. the mass extirpation of on- 
chocercal nodules. However, chemotherapy studies must also be encouraged.

\section{(4) Health education}

The health education of people living in endemic communities is important to promote the smooth execution of field control activities. To that end, for this purpose, existing section in charge of health education in the Ministry of Health and Welfare will be beneficial to the project (quotation source No. 3, p. 4).

(Chapters 2-3 to 2-6 were not cited)

\section{Results and Problems Encountered In the First Five Years}

The following is a review of the results of the first five-year phase starting in October 1975, as well as the problems pointed out in the evaluation team's report (quotation source No. 9).

\section{3-1 Results and evaluation by the epidemiology and parasitology sections}

The duties of the epidemiology and parasitology sections in the first five-year phase starting in October 1975 can be divided into the following three main goals: 1) to elucidate the epidemiological characteristics of onchocerciasis in San Vicente Pacaya (SVP), the pilot area; 2) to evaluate the effect of vector control; and 3) to develop techniques necessary to achieve the above two goals and to conduct basic studies on onchocerciasis.

In view of these basic policies, the results and accomplishments of the past five years can be considered mostly satisfactory.

Various obstacles and limitations including the short duration of the project, the shortage of staff (particularly the counterparts), and the limited cooperation of inhabitants, it was necessary to emphasize goal (1) as the first and goal (2) as the second priority. Most of the plans related to the fundamental research of goal (3) were left unresolved.

The biggest disappointment was the fact that, due to the delay in vector control, the evaluation mentioned in goal (2) was not implemented during the period, and effective evaluation methods could not be sufficiently developed.

The following is a review of individual matters.

\section{(1) Epidemiological characteristics}

The SVP pilot area stretched over the southern slope of the Pacaya volcano, an area of approximately $250 \mathrm{~km}^{2}$ between the altitudes of 300-1800 m. It included the town of San Vicente Pacaya located in the northernmost part and dozens of other villages and coffee plantations. The population was approximately 6,000 people, half of which lived in SVP. The infected population was relatively low.

Since the inhabitants of the town were uncooperative, as is customary in studies of this sort, the survey was not sufficiently conducted except in La Cruz located in the west part of the town with a relatively high prevalence. However, half of the population or approximately 3,000 from other areas were successfully examined.

The inhabitants were investigated systematically and precisely as follows. For the epidemiology investigation, parasitologic detection of the microfilaria by skin biopsy was adopted. For the clinical investigation, nodules, dermal and ophthalmological, particularly microfilarial, signs were checked. The immune diagnoses included skin test, indirect hemagglutination (IHA), and double diffusion. In some cases, fecal examination was also performed. Not all of the examinations were applied to every inhabitant, but at least the nodule examination covered the necessary size of the population.

The purpose of the epidemiology investigation consisted of the following two goals: 1) to grasp the epidemiological characteristics in the area and 2) to obtain baseline data for comparison with the post-control data. The results are as follows:

1) To grasp the epidemiological characteristics in the area

In the SVP pilot area, the microfilaria-positive rate was approximately $40 \%$, a medium prevalence in the country. However, the value fluctuated from 0 to $90 \%$ by village, and the higher prevalence was seen in villages located at an altitude between 600 and $1300 \mathrm{~m}$ in the northwestern part of the area. The epidemiological evidence was useful in identifying the main vector as $S$. ochraceum by entomological investigation and in initiating the vector control in this topographical area.

Clinical investigations revealed that more than half of the onchocercal nodules were situated on the head and that the microfilarial density was higher in the upper body. Although characteristic for Central America, these findings differed markedly from the clinical features of African onchocerciasis. Another finding was that the dermal and ophthalmological lesions were rather mild in comparison with African lesions. The rate of blindness apparently caused by onchocerciasis was also low, showing only about $0.2 \%$. We also analyzed the sex and age-specific characteristics of infection and the relationship between working/inhabiting places and breeding areas of the vector fly. Further important epidemiological findings were obtained as to the microfilaria rate, nodule rate, and immune reactions related to clinical signs. Although not fully analyzed, the existing data were systematically recorded on individual cards that 
could be used over the extended period for computer analyses.

One remaining question is the elucidation of the socioeconomic burden of onchocerciasis on the coffee plantations that are frequent in the area and constitute the major industry of Guatemala. This should be analyzed in the future.

Unfortunately, very few records were taken as to the vector density in individual villages, in spite of the fact that the prevalence of onchocerciasis in individual villages was clearly shown. The minimum essential vector density necessary for the transmission should have been assessed in terms of annual biting density per person by the analysis of transmission dynamics. This point should be assessed over the extended term.

2) Collection of baseline data

The epidemiological characteristics of prevalent disease and individual records obtained by the above epidemiological survey comprise the baseline data. Since the purpose of the project is not the elimination of vector blackflies but the control of onchocerciaisis, the final evaluation should be conducted on the inhabitants. Therefore, the collection of baseline data was the most important task for the epidemiology/parasitology section.

In this project, however, the policy of vector control was to reduce vector blackflies by larvicidal measures at the breeding sites. Considering the long lifespan of the parasites in humans, it may take some time before statistical differences emerge between the baseline data and postcontrol data. The earliest sign of success in the vector control will be a reduction in the microfilaria rate or negativity of microfilaria carriers among children, particularly those born after the vector control. Even in areas where the vector control started in March 1979 at the end of the final stage of the first five-year project, about four years were required for the evaluation in 1983, the final year of the second project period. In this sense, the examination of children below four years, or if possible two years of age, will be necessary. Unfortunately, the data were extremely lacking in this age bracket because of the difficulty of performing skin biopsies. This point should be addressed in the second stage of the project.

\section{(2) Evaluation of the effect of the control}

There are two ways to evaluate the effect in the human population, one a long to medium-term evaluation expressing the effect after a long period, the other a shortterm evaluation expressing the effect even after a short period. As the project was conducted within a short period of time, the latter evaluation was preferable.

In the first five-year phase of the project, the most important issues in the epidemiology/parasitology section were the development of evaluation measures and their application in the field. The evaluations were not performed during this period because the control trial itself was delayed but are expected to be accomplished in the extended period.

In the first five-year phase, however, preparations are being made for a short-term evaluation. To show the effect of control, the basic strategy is to assess the incidence between two time-points, not the prevalence at a determined point of time. Pre-control incidence in the endemic areas constitutes an important baseline data. The following is a summary of the baseline data in the first five years.

1) In the second project phase, the streams in five villages (Canton de la Cruz, Patrocinio, Los Rios, Finca Guachipilin belonging to Barretal and Guachipilin) were selected as experimental areas, while Finca Hamburgo, which was beyond the influence of vector control, was selected as the control site. Unlike conventional epidemiological surveys to obtain baseline data, the sites were chosen because of their convenience for periodical epidemiological surveys at determined intervals, thus causing a decrease in the sample population.

2) Precise epidemiological surveys were planned at intervals of exactly one year in the above villages. The initial idea had been to repeat them at six-month intervals in view of the wet and dry seasons. This plan was rejected due to the shortage of staff and other limitations. In fact, even the examinations at 12-month intervals were difficult to perform. Revision will be necessary in the process of analysis.

3) The following three items were adopted in assessing incidence

a. Positive conversion rate by skin biopsy

b. Appearance of onchocercal nodules

c. Positive conversion or an increase in wheals in immune reactions (skin test, IHA, DD)

Ophthalmological findings (particularly punctate keratitis) and skin lesions (particularly biting mark) were considered important as well. To determine the positive conversion, the subjects previously examined should be selected. Along with the improvement of sero-diagnosis techniques, it is important to carefully apply the methodology and antigens etc. used in the baseline data for precise comparisons during the observation period.

\section{(3) Fundamental research}

In the first five-year phase, together with the extended three-year phase, the potential of disease control was demonstrated by vector control and the related evaluation was emphasized in the study. Therefore, it was necessary to develop specific and simple diagnostic methods to obtain ep- 
idemiological data in the epidemiology/parasitology section, a purpose that was successfully achieved.

With regard to skin biopsies, for example, the use of Holth-type corneo-scleral punch produced excellent results. As to the biopsy site, it was concluded that the shoulder and loin in men and the shoulder in women were the most preferable. Antigens extracted from both the adults and microfilariae of $O$. volvulus showed high specificity and sensitivity in the immunological diagnosis. These findings efficiently supported the epidemiological and parasitological surveys.

On the other hand, various fundamental studies could not be performed due to the shortage of time and staff. In the study on techniques of immune diagnosis, the comparison of specificity/specificity was not performed. Furthermore, the influence of other species of filarial, helminthic and protozoal infections was not examined.

Interesting findings were obtained regarding the occurrence and size of onchocercal nodules. In the field of parasitology, the infection process and growth of $O$. volvulus L3, the production mechanism of microfilariae, the pathogenicity of onchocercal lesions and the involvement of immune state are exciting topics for researchers because they have not been fully investigated worldwide.

The necessity for chemotherapy studies was described in the first and second phases, but they were left for the future because of their irrelevance to onchocerciasis control. The development of chemotherapy should be promoted to eliminate the source of onchocercal infection, but since the Japanese specialists were not permitted to engage in treatment, their role was limited to supporting and encouraging the Guatemalan staff in clinical activities. Accordingly, further efforts are needed to encourage the Guatemalan side in the extended plan.

On the other hand, animal experiments using the mouse-microfilaria model were encouraged. Microfilariae and even nodules containing adult parasites were inserted into mice to examine the behavior of the adult parasites. Although the adults soon died, the microfilariae dispersed and gathered in the auditory capsule and tail, surviving for at least two weeks. Diethylcarbamazine (DEC) and other antifilarial agents were administered experimentally to the infected mice. The importance of this type of animal experiment was recognized in the project. $O$. volvulus is able to infect only humans and chimpanzees. For this reason, a convenient experimental animal model is urgently required. The followings are the reasons.

1) Research on the infection and growth of $O$. volvulus

There are many unknown issues regarding larval growth, migration, nodule formation and isolated parasitism etc. in the body of the definitive host. Similarly, the in- tracorporal distribution and migration of microfilariae in the skin should be investigated.

2) Pathogenicity studies

Pathogenicity studies were conducted to elucidate the mechanism of the ophthalmological and dermal lesions. The injection of microfilariae directly into the anterior chamber of rabbit eyes was attempted in the first five-year phase, and the migration of injected microfilariae was observed in the corneas. Further experiments are necessary using experimental models.

3) Development of chemotherapeutic agents

In order to obtain agents effective for microfilariae, as well as juvenile and adult onchocerca, an experimental animal is necessary like the chimpanzee, which harbors all stages of $O$. volvulus. At this moment, any animal small in size and cheap that is available to harbor microfilariae, juveniles and adults, will be acceptable for the screening studies (quotation source No.9, p. 7-11).

\section{3-2 Results and evaluation of the entomology section (1) Determination of vector species}

The conventionally recognized vectors of onchocerciasis transmission in Guatemala are $S$. ochraceum, $S$. metallicum, S. callidum, but $S$. ochraceum has been emphasized recently as the main vector.

Even in this project, the confirmation of target species was started in the first year in order to avoid a decisive failure in the control due to a wrong target. The study of more than one year identified only S. ochraceum as the target, although the role of S. metallicum and S. horacioi was suspicious, making $S$. ochraceum the only realistic target for the control trial.

\section{(2) Ecology of the vector}

1) Larvae

The larvae of the vector $S$. ochraceum breed in small shallow streams of $0.1-10$ liters/second located at the source of the water system as if licking the earth's surface. Since, the identification of these streams was vital to the larvicidal vector control, studies on the development of search methods, the identification of characteristic breeding streams, and the examination of the larval ecology including the growth period were performed.

2) Adults

With regard to adult flies, the bloodsucking activity, etc. was investigated mainly to clarify the transmission mechanism. Studies on seasonal changes in bloodsucking activities, host preference, preferable body portion for biting and gonotrophic cycles were the main themes.

3) Transmission mechanism

Elucidation of the transmission mechanism, an interesting topic in itself, was an important aspect of efforts to 
promote research on two points in the project. One was the need to select the vector. To this end, investigations were conducted on natural infection and the transmission activity in experimental infection. The other was the decision as to the threshold at which the transmission is halted by lowering the vector density. The target value for the vector control, this numerical value was found to be 15.3 flies/day per human bait.

Furthermore, as factors characterizing the distribution of disease, the relationship between altitude and temperature and the growth of onchocercal larvae was clarified in the blackfly, along with the relationship between bloodsucking time and the number of ingested microfilariae.

\section{(3) Examination of vector control}

Both the work to determine breeding places and the aerial spray of insecticide were hampered by the precipitous topography of Guatemala. Spraying the larvicides on the ground was the only possible method of vector control. Thus the insecticide formula and application method were studied along with the interval between sprays. Slowrelease agents of 5\% Temephos (Abate) wettable powder hardened with surfactant were placed in the streams once every two weeks. The preparation of maps of the water systems and insecticide application was a prerequisite for efficient operation and precise control. For this purpose, in addition to the detailed maps drawn by the groundwork, a geological methodology was developed to disclose breeding sites.

\section{(4) Vector control operations}

As a first step, insecticide treatment was started in the water systems of Lavaderos from March 1979. As a second step, treatment was started in the Barretal/Zapote water systems in June the same year. The application of insecticides continued once every two weeks and continues today. For the evaluation, breeding blackfly larvae were examined just after the insecticide treatment, and finally the density of adult blackflies was assessed using human bait collection.

As a result, a marked decrease in blackfly density was observed in the basin of the above water systems at present, raising hopes for a halt to disease transmission. The inhabitants were happy to be free from blackfly bites. At this moment, the mapping operation in the Guachipilin streams has been completed as the next stage, and the preliminary application of insecticides has started. The fiveyear phase will end with the insecticide treatment of the Guachipilin water systems (quotation source No. 9, p. 1516).

\section{3-3 Problems encountered in the first five-year phase}

A vector control strategy was adopted in this project because of the absence of chemotherapeutic agents for mass treatment of the inhabitants aimed at eliminating onchocerciasis. It may be appropriate to apply larvicides to blackfly larvae in localized spots at this stage. For this operation, detailed mappings were accomplished and the proper dosage and formulation of insecticide were determined by fundamental research. Finally, periodical insecticide treatment was started in March 1979 in the Lavaderos ravine belonging to the Barretal water system located in the northernmost region of the pilot area. Approximately one year later, in May 1980, the evaluation team did not find any larvae in the treated streams or blackfly adults by human bait collection. This remarkable result won high international commendation, praise from the government of Guatemala and the appreciation of the inhabitants.

The goals of the project were not all achieved in five years, but the results provided clues for further vector control of the Central and South American types of onchocerciasis. Considering the limited time of only five years and the shortage of staff, the work proceeded with remarkable speed in the absence of fundamental research (quotation source No. 9, p. 3).

In the epidemiology/parasitology section, the development of effect-assessment techniques and the inspection of effectiveness were not performed because of the delay in the schedule of vector control. Furthermore, the entomology section was unable to sufficiently address the following four points due to the time limitation.

\section{(1) Problems related to the vector}

The target has been narrowed down to $S$. ochraceum alone at present, but $S$. metallicum and $S$. horacioi remain suspicious. Even if they are apparently inferior to $S$. ochraceum in the role of vector, the experimental infection showed a high potential for transmission. Furthermore, even though the frequency was extremely low, natural infection was observed in those species. If S. ochraceum disappeared as a result of vector control, the other species could emerge as alternative vectors for transmission. This possibility needs to be noted, but in any case more studies are necessary on the role of $S$. metallicum and $S$. callidum.

\section{(2) Flight range of the adult blackfly}

An understanding of the flight range of the vector blackfly is a prerequisite for vector control and epidemiological decisions. Although the priority was rather low, the project included a plan to investigate this issue. However, the investigation was not performed because it demanded enormous manpower and time. In the initial stage of vector control in Barretal, the reduction of adults was not satisfactory. The detection of previously overlooked breeding sites and the larvicidal operations there somewhat improved the result, but not as much as in Lavaderos. This finding is 
probably attributable to the invasion of adult blackflies from Rodeo, an untreated region located in the southern portion of the pilot area. In any event, therefore, the postponed research on flight range should be started.

\section{(3) Formulation of the insecticide}

At first, Temephos (Abate) hardened with beef fat and polyvinyl alcohol was used as the larvicide. This method was chosen to ensure the slow release of the insecticide over a period of about two hours and to facilitate formulation, handling and weighing etc. However, considerable disparity was noted in the insecticide-releasing time, and the raw materials were difficult to acquire at the local market. After several trials, Temephos (Abate) wettable powder hardened with a surfactant was adopted for the control. However, the production was still insufficient, and further improvements were required.

\section{(4) Vector density precluding transmission}

The purpose of vector control is to reduce the density of the vector blackfly to a level low enough to preclude transmission. Since this is essential, the setting of ABR and ATP, which have been used in the OCP of West Africa, was necessary.

As a temporary criterion, the level of 15.3 fly/day/ person was used in this project, but a more extensive investigation is necessary to raise the numerical reliability (quotation source No. 9, p. 17).

(Chapter 4 was not cited)

\section{Results and Evaluation of the Extended Three-year Phase}

This project, which started with a five-year phase, was extended an additional three years in 1980 to offset the delay in the general plan. The major reason for the delay was that the control operations did not advance as expected. The initial plan called for control operations to start in all areas in 1979, but in fact they started only in Lavaderos, Barretal and Zapote located in the northeast extremity of the pilot area and accounting for only $9.9 \%$ of the target area. In the additional three years, the area of operation was enlarged to include Guachipilin (1981), Jasmines, Chilcas, Conacaste (1982) and Rodeo (1983). These additional areas accounted for approximately $38.1 \%$ and brought the total covered area to $60 \%$ of the initial plan.

Generally speaking, research tends to be endless in nature, making it difficult to arrive at immediate conclusions. Since the project progressed without sufficient arrangements, the following issues remained unresolved.

\section{(1) Species complex analysis of the vector blackfly}

To date, the main vector Simulium ochraceum has been regarded as a single species. But several observations suggest that this species is a species complex, a hypothesis that can be confirmed not only by morphological data but also cytogenetic studies on chromosomes and biochemical studies on isozymes. Although some trials were started, they ended without finishing due to lack of time.

\section{(2) Studies on the transmission mechanism}

The vector control of the project targeted only $S$. ochraceum, but evidence has appeared suggesting that this was not the only blackfly species involved in transmission. The role of many auxiliary species such as S. metallicum and $S$. horacioi remains unclear, particularly the substitute species possibly emerging after the elimination of $S$. ochraceum.

\section{(3) Ecology of the vector}

The precise details of larval and adult S. ochraceum have not been fully demonstrated yet.

\section{(4) Examination of the vector control method}

In the project, $24 \mathrm{~g}$ Temephos (Abate) wettable powder was applied ubiquitously. However, Temephos shows a high rate of adsorption to the substratum in water and the loss of effect is strong. The formulation and application procedures should be improved. In addition, the development of substitute insecticides must be considered in preparation for the time when the blackfly acquires resistance to Temephos.

\section{(5) Differentiation of the genus Onchocerca}

The taxonomical identification of larvae of the genus Onchocerca remains impossible and constitutes a serious obstacle to research.

\section{(6) Development of chemotherapy}

For various reasons, the Japanese specialists did not participate in the treatment and clinical study, and little progress was made in this aspect of the project.

As mentioned above, many issues remain unsolved likely left-off cargos in the research field, but looking at the project over its extended period of eight years, it is reasonable to conclude that the initial goals were mostly achieved.

Fig. 2 shows the annual biting density of $S$. ochraceum. In Lavaderos, where the vector control started in 1979, the annual reduction was remarkable, and when the project ended in 1983, the density had decreased to one one-hundredth the pre-control level. 


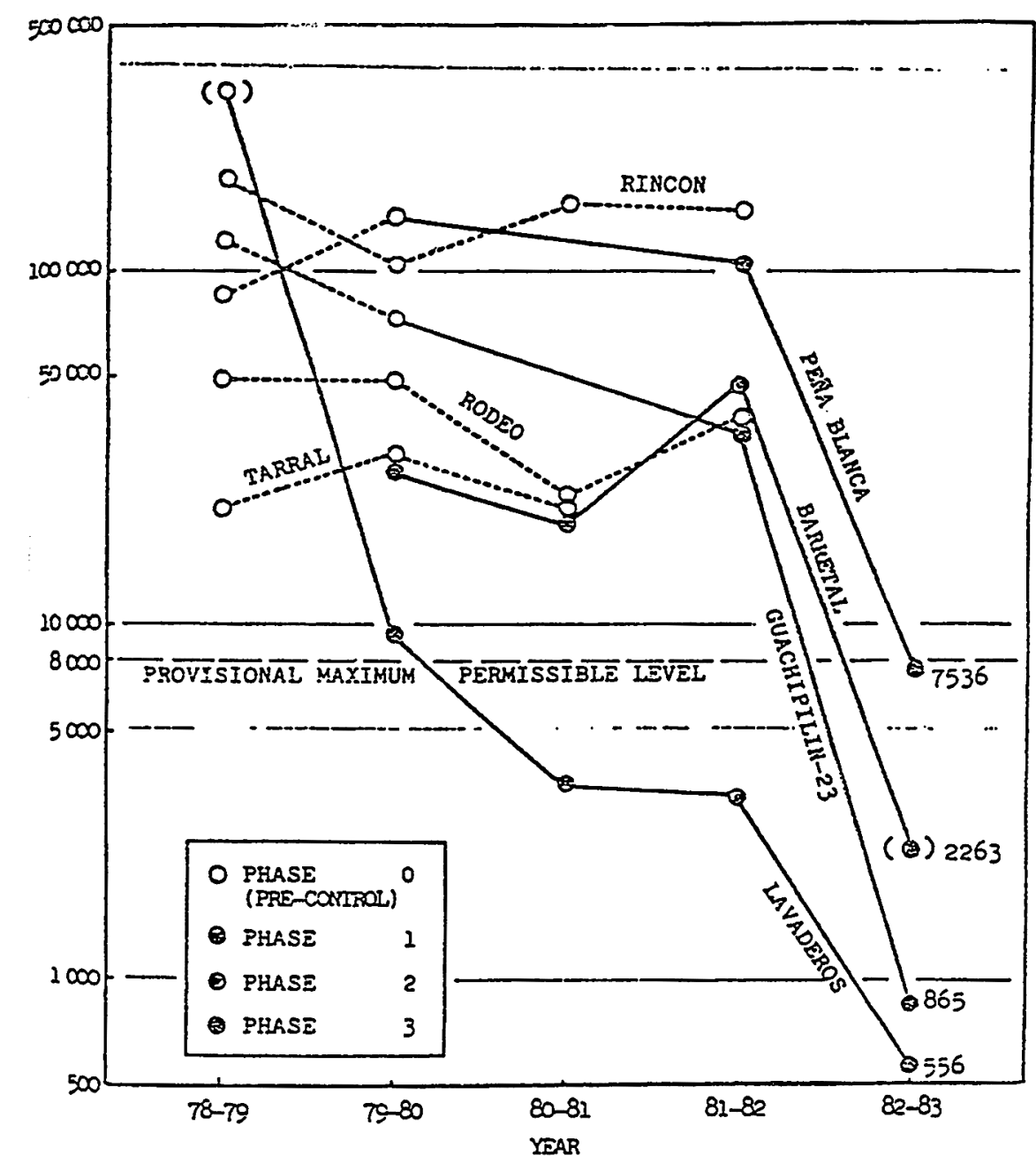

Fig. 2. Entomological evaluation of vector control operation in SVP: The ABR in relation to the control phases ( ): Covering 810 months only. (Yamagata et al., Series No. 100)

Even in other areas, a remarkable reduction was observed after one year, and the vector density was lower than the level of possible transmission. These facts indicate that the project accomplished the control of the vector blackfly in Guatemala and central American countries.

The following is a list of manuals and textbooks based on the information and techniques gained from the project.

A. A Guidebook for Guatemalan Onchocerciasis (Robles disease). pp. 155. 1983

B. Manual of Onchocerciasis (Robles disease) Control in Guatemala. pp. 162. 1983

C. Glossary of Technical Terms Related to Onchocerciasis (Robles disease) in Guatemala. pp. 93. 1983

D. Libro de Texto Basico para los Estudios de la Oncocercosis en Guatemala. pp. 170, 1983

These manuals and textbooks provide necessary guid- ance on the methods useful for the control programs expected to be implemented throughout Guatemala.

The final target of the project, they are highly valuable in themselves.

During the project, areas remained where no attempts were made to reduce vector density in the planned pilot area or to evaluate the effect of vector control in the human populations. In retrospect, however, that situation may be regarded as unavoidable.

The manuals may contribute to vector control not only in Guatemala but also in other South/Central American countries. At present, they serve as standard textbooks in the field and are expected to elicit the interest of WHO and the African countries concerned (quotation source No. 17). 


\section{Comprehensive Evaluation of the Project}

\section{6-1 Comparison of the plan and the results}

An outline of the eight-year project (five years plus a three-year extension) is shown in Tables 3 and 4. A comparison of the implementation plan and the achievements of the eight-year project are shown in Tables 5 and 6. Table 7 shows the overall achievements including the investigation team, the dispatch of specialists, equipment donation and the acceptance of trainees over the entire period of the project (quotation source No. 17).

\section{6-2 Comprehensive evaluation of the epidemiology/ parasitology field}

(1) To obtain baseline data before control operations, the prevalence of onchocerciasis was determined in the pilot area (San Vicente Pacaya, with approximately 6,000 inhabitants) on the basis of the microfilarial rate obtained from skin biopsies, the frequency of nodules, skin and ocular manifestations and the rate of positivity in skin tests and IHA etc. The analysis of these data revealed the epidemiological features of distribution and prevalence by sex and age and the association with breeding places.

(2) Social unrest hampered investigations in other endemic foci in Guatemala, and in most cases only a rough survey was conducted.

(3) Studies to simplify and improve the specificity of various diagnostic procedures like skin biopsy, skin test, IHA, DD and ELISA were carried out successfully together with the improvement of antigen extraction.

(4) Studies on the mechanism of nodule formation and ocular manifestations conducted through animal experiments revealed important findings regarding the pathogenicity of onchocerciasis.

(5) However, many issues related to the pathological process of ocular disorders and dermal lesions remain unresolved.

(6) The criteria to differentiate the nematode larvae similar to Onchocerca volvulus larvae found in the body of blackflies caught in nature were not established during the project period.

(7) Efforts to develop a useful laboratory animal model to study the pathogenesis of onchocerciasis and chemotherapeutic agents were started but did not reach completion.

(8) Research on chemotherapy in humans was the responsibility of physicians on the Guatemalan side. Guidelines for DEC administration were established tentatively, but Mebendazole, a bezimidazole agent, could not be tested in spite of its promising features.
(9) An annual epidemiological survey was carried out continuously in the pilot area (SVP), and the necessary technology was almost completely transferred. It was marvelous to see the Guatemalan side handling the work alone. Today, the Guatemalan staff is singlehandedly accumulating the valuable data needed to judge the effect of vector control.

(10) Unfortunately, since the vector control depends on insecticide spraying in the breeding streams, no data were obtained by the end of the project to determine the effects on onchocerciasis transmission in the human population living in endemic areas. Since the earliest operation started in March 1979 and the latest in January 1983, there was not enough time to confirm the effect (quotation source No. 17).

\section{6-3 Comprehensive evaluation of the entomology field}

(1) One of the great achievements of the project was the identification of the blackfly fauna in and near the pilot area (SVP) in Guatemala. Studies on the species and distribution of blackflies were intensively performed. Notably, the description of a new species and non-recorded species by Japanese scholars was included with the introduction of cytotaxonomic and isozymic studies, stimulating great academic interest in Guatemala.

(2) Findings on the distribution of blackfly species in SVP as well as the prevalence and the distribution of onchocerciasis in humans indicated that the main vector was $S$. ochraceum.

(3) Studies on the major anthropophilic blackfly species, the degree of anthropophilic behavior, the growth of $O$. volvulus larvae in the body by experimental infection further indicated that $S$. ochraceum was the main vector in Guatemala. The findings helped to limit the geographic scope of vector control operations.

(4) However, various biological and ecological studies on blackfly species were left unfinished.

(5) A tentative standard was established for the formulation, dosage and application of insecticide suitable to the streams where $S$. ochraceum breeds in Guatemala. These results facilitated the efficient application of insecticide in the breeding areas under difficult conditions. Further studies are necessary, and the insecticide susceptibility should be monitored consistently in the future.

(6) Transmission studies on the blackfly showed that, in order to maintain transmission, the required annual biting by the vector species was approximately 8,000 flies per person/year. This value provided the tentative index necessary to evaluate the effects of control 
measures.

(7) A detailed map was prepared to chart the water systems in SVP and to show the breeding sites for $S$. ochraceum. It should also be noted that the technology necessary for the mapping of water systems and vitally important for vector control was transferred successfully.

(8) To determine the effect of control operations, a method to measure the inhabitation density of $S$. ochraceum was established. Implemented at fixed points to periodically measure the larval density in the water and the adult density using human bait, this method was developed in such a way as to be applicable to the endemic areas of Guatemala.

(9) In each area of SVP where the above larvicidal operation was carried out, for example Lavaderos, 300-400 S. ochraceum attacked one person over a three-hour period in the morning before the control operation. This number was reduced to less than ten after the control, showing a number far lower than 8,000 flies a year. Similar results were later obtained in the water systems in the extended area (quotation source No. 17).

(Chapters 6-4 to 6-5 were not cited)

\section{6-6 Lessons and Proposals}

\section{(1) Planning}

Since the period of cooperation in research projects tends to last for the long term, the initial target should be kept to a manageable size. In the present project, the pilot area was so large that almost one-third of it remained unexamined.

\section{(2) Implementation of the project}

The Japanese specialists came from various parts of the country, making it difficult to coordinate the communication of instructions to counterparts and to select the equipment to be donated.

In view of technology transfer, the domestic steering committee should be able to exercise leadership in the methodology and the choice of equipment. Limitations faced by the domestic supporting institutions should also be considered.

\section{(3) Evaluation of activities}

The evaluation from the Japanese side was conducted efficiently thanks to cooperation among the dispatched specialists. Unfortunately, however, the evaluation from the Guatemalan side was not sufficient. The project should require the Guatemalan side to submit an annual report on the evaluation and results.

\section{(4) Remaining questions}

The expiration of the cooperation period mentioned in the R/D should not mean an immediate end to the cooperation but should call for the dispatch of a single specialist. The decision in the present project to continue to dispatch specialists after the expiration of the project precluded any trouble on the point.

The following is a list of issues left unresolved at the time of the project expiration.

1) Parasitology/epidemiology

a. Improvement of parasitological and immunological diagnosis

b. Clarification of the systematic differences of $O$. volvucus class

c. Clarification of the biological and biochemical features of $O$. volvulus

d. Elucidation of the mechanism and pathogenicity of various symptoms

e. Chemotherapy research

f. Development of a laboratory animal model

g. Development of epidemiological techniques to assess the effect of vector control and mass treatment

2) Entomology

a. Elucidation of vector blackfly species, particularly $S$. ochraceum complex

b. Biological and ecological studies on potential vector blackfly species and their distribution, population density, growth velocity, seasonal prevalence, compatibility with $O$. volvulus, anthropophilic character, biting behavior, life span, flight range etc. The relationship between these features and environmental distribution should be clarified.

c. Studies on insecticide susceptibility and related changes

d. Improvement of vector control methodology

e. Transmission dynamic studies on onchocerciasis.

f. Studies on indoor blackfly breeding and the establishment of experimental colonies

Although some of these questions were studied to some extent during the project period, most of them were left uncompleted. They should be addressed in the future considering their importance.

(Chapter 6-6 (5) was not cited)

\section{6-7 Evaluation at one year after the project expiration}

In projects involving overseas technical cooperation, there are many cases in which continuation is hampered by limitations of financial support, talented personnel and equipment in the recipient country when the project ends. In the case of this project, however, there was no such a 
problem, the Guatemalan side actively continuing the onchocerciasis control.

Three of the Japanese specialists, Takeshi Suzuki (team leader), Mamoru Ito (epidemiology/parasitology) and Sumiyo Ito (entomology/vector control) reported as follows one year after the expiration of the project in Guatemala (May to August 1984, quotation source 15). They had been dispatched to Guatemala in the second three-year project as long-term specialists.

\section{(1) Epidemiology and parasitology}

The epidemiological investigation of prevalence throughout Guatemala progressed smoothly. A new system in which each survey team took charge of specific areas was adopted. This system was conducive to managing the responsibility of each team and establishing friendly relationships with the local inhabitants.

This kind of epidemiological investigation provides the basic data necessary for future nationwide controls. It is understandable therefore that Guatemala places emphasis on this investigation. Furthermore, the active investigations presently underway deserve appreciation and recognition.

Mass treatment with DEC is carried out at two fincas (plantations). Although progress is smooth, it is considered to be just a trial at this stage. In order to introduce this measure into the control, various issues such as the participation of physicians in the mass treatment should be taken into account.

\section{(2) Entomology and vector control}

The vector control area was expanded from $55.2 \mathrm{~km}^{2}$ in late September 1983 to the current $91.3 \mathrm{~km}^{2}$, an increase of $65 \%$. In these areas, the density of the main vector $S$. ochraceum has been reduced to an extremely low and satisfactory level. For the future expansion of vector control, preliminary surveys have already been completed in
Hamburgo $\left(44 \mathrm{~km}^{2}\right)$ and Yepocapa $\left(9 \mathrm{~km}^{2}\right)$. Further improvements were seen in mapping techniques and staff training.

(Chapters 6-7 (3) to 6-7 (5) ware not cited)

\section{6-8 Reasons for the success of the project}

The project was unexpectedly successful in the vector control of onchocerciasis in Guatemala, a task thought to be extremely difficult. The reasons for this success are discussed briefly at the end of the book. The contents are cited from a review by Y. Yamagata, who remained in Guatemala for a follow-up study after the end of the project (quotation source No. 18).

According to him, the success of the project is attributable to the special skills of the staff from both sides. The Japanese side was mainly in charge of fundamental research and tactics development, while the Guatemalan side handled countermeasures. The vector control would have failed without both contributions. The two sides complemented each other in research and operation.

In simple terms, vector control is a war against the vector insect. As well as skillful tactics and powerful weapons, well-trained and organized battalions are necessary. When the project started, the Japanese specialists had neither experience in actual fighting nor awareness that vector control was tantamount to war. In other words, the Japanese specialists were totally unprepared for combat. Luckily, however, the Japanese side enjoyed the cooperation of SNEM in Guatemala. SNEM had a history of 30 years and a staff of 900 people who had made excellent achievements in the vector control of mosquito against malaria, dengue fever, yellow fever, etc. Their accumulated ability could be utilized in the vector control of blackflies. 
7. InPUt COST From JAPAN By Fiscal YeAR

\begin{tabular}{lrl}
\hline Year & $\begin{array}{r}\text { (units of } \\
1000 \text { yen })\end{array}$ & \multicolumn{1}{c}{ Contents } \\
\hline 1973 & 1,496 & 1 specialist \\
1974 & 4,171 & 4 specialists in a preliminary investigation team \\
1975 & 10,571 & 4 specialists in a preliminary investigation team and equipment including microscope \\
1976 & 92,039 & 12 specialists and equipment including lyophilizer etc. \\
1977 & 157,394 & 24 specialists in total including a programming team and equipment including vehicles, microscope etc. \\
1978 & 137,680 & 26 specialists in total including evaluation team and equipment with microscopes, incubators etc. \\
1979 & 113,906 & 9 specialists and equipment including deep freezers, EEG etc. \\
1980 & 139,972 & 39 specialists including evaluation team \\
1981 & 143,814 & 8 specialists \\
1982 & 8,547 & \\
1983 & 9,916 & evaluation team \\
\hline
\end{tabular}

(modified from original table style)

(Anex-1 to 3 were not cited) 